\title{
Análisis interdisciplinario del estado actual de la cultura ambiental desde los componentes técnico, social, comunicacional y pedagógico. La universidad y su apuesta al desarrollo sostenible*
}

\author{
Ana Lorena Malluk Marenco* \\ Flor Delgado Sánchez ${ }^{* * *}$ \\ Rafael Figueroa Altamiranda****
}

Recibido: 2016-04-26. Enviado a pares: 2016-05-05

Aprobado por pares: 2016-05-28. Aceptado: 2016-06-10

DOI: 10.22395/angr.v15n29a7

\section{Resumen}

El artículo plantea los desafíos que trae consigo el análisis del estado actual de la cultura ambiental; más aún cuando la universidad es considerada la institución divulgadora de conocimiento y formadora de ciudadanos promotores de ideas, decisiones y comportamientos que contribuyan al desarrollo ambiental sostenible.

Se caracterizaron las realidades ambientales que dan origen al escenario actual, para a partir del aporte de los grupos de interés, iniciar el proceso de construcción del escenario posible y deseable. El análisis documental, la revisión de los planes de estudio y la encuesta fueron las estrategias metodológicas que aportaron a la documentación teórica, la interpretación de evidencias y la interacción de los investigadores con los actores y sus realidades.

Los resultados evidenciaron que en la Universidad Pontificia Bolivariana, seccional Montería, la relación hombre-sociedad-naturaleza se ve afectada por el uso y ahorro de materias primas, energía y agua; manejo de residuos sólidos y líquidos; aprovechamiento de zonas comunes; análisis ambiental en los planes de estudio; apropiación del ciudadano; uso de la comunicación para generar procesos de transformación social, y desarrollo de proyectos de investigación y de iniciativas de proyección social en materia ambiental.

Palabras clave: cultura ambiental, sociedad, universidad, educación, comunicación.

\footnotetext{
- Este artículo es resultado del proyecto de investigación interdisciplinario "Análisis del Estado actual de la cultura ambiental de la Universidad Pontificia Bolivariana Seccional Montería" financiado por la Universidad Pontificia Bolivariana Seccional Montería (Radicado CIDI 163-04/13-G012). (COLOMBIA).

** Comunicadora social-periodista; magíster en Comunicación; Grupo Comunicación y Educación COEDU; Escuela de Ciencias Sociales y Humanas; Universidad Pontificia Bolivariana; ana.malluk@upb.edu.co.

*** Psicóloga; magíster en Educación; Grupo Calidad de Vida CAVIDA; Escuela de Ciencias Sociales y Humanas; Universidad Pontificia Bolivariana; Seccional Montería; Colombia. flor.delgado@upb.edu.co

**** Licenciado en Ciencias Sociales; magíster en Proyectos de Desarrollo Social; Escuela de Ciencias Sociales y Humanas; Universidad Pontificia Bolivariana; Seccional Montería. rafael.figueroa@upb.edu.co
} 


\title{
Interdisciplinary Analysis of the Current Status of Environmental Culture from Technical, Social, Communicational and Pedagogical Components. The University and its Commitment with a Sustainable Development
}

\begin{abstract}
This article poses challenges that the analysis of the current status of environmental culture brings; even when the university is considered the institution for disclosing knowledge and educate citizens who promote ideas, decisions and behaviors contributing to the sustainable environmental development. Environmental realities which originate the current arena were characterized, so from the contribution of interest groups, the construction process of a possible and desirable arena may be started. The documental analysis, revision of courses of study and the survey were the methodological strategies, which contributed to the theoretical documentation, the interpretation of evidences, and the interaction of researchers with actors and their realities.

Results evidenced that in Universidad Pontificia Bolivariana, Montería campus, man-society-nature relation has been affected by the use and savings of raw materials, energy, and water; solid and liquid residues handling; use common zones; environmental analysis in courses of study; appropriation of the citizen; use of communication for generating social transformation processes, and development of research and social projection projects in environmental issues
\end{abstract}

Key Words: environmental culture, society, university, education, communication 


\section{Introducción}

El progresivo deterioro que experimenta la sociedad actual se atribuye a valores, creencias y actitudes ambientales que generan determinados comportamientos o conductas ecológicas, lo que en conjunto determina la cultura ambiental. Frente al uso exacerbado e irracional de los recursos naturales, académicos, investigadores y expertos estudian los fenómenos asociados al problema y avanzan en la construcción de escenarios que contribuyan al desarrollo ambiental sostenible y, por ende, al mejoramiento de la calidad de vida.

Si la cultura es un término globalizador que incluye todo aquello que la humanidad ha incorporado en la naturaleza - con el fin de dominarla, transformarla, establecer relaciones sociales acertadas, generar respuesta a sus interrogantes y tener su propia cosmovisión (Beldarrín, 2004)-, el ser humano es portador de valores culturales. Se comprende así la cultura ambiental como la forma en que las personas se relacionan con el ambiente; de ahí que su tratamiento implique la necesidad de un enfoque educativo y cultural, que se aborde desde los valores, creencias, actitudes y comportamientos ecológicos (Bayón y Morejón, 2005).

La Organización de las Naciones Unidad-ONU- plantea la importancia de realizar una labor de educación en cuestiones ambientales para ensanchar las bases de una opinión pública bien informada y de una conducta de los individuos, de las empresas y de las colectividades inspirada en el sentido de su responsabilidad en cuanto a la protección y mejoramiento del medio en toda su dimensión humana (Programa de las Naciones Unidas para el medio ambiente, 2005).

En vista de que la cultura ambiental es un tema amplio, complejo y objeto de intervenciones de diferentes disciplinas y actores, la Universidad Pontificia Bolivariana define dentro de los elementos fundamentales de su filosofia la antropologia cristiana, que propende por la íntima relación hombre-naturaleza (Universidad Pontificia Bolivariana, 2005); la importancia de fortalecer la línea Entorno-generación de procesos interdisciplinarios de investigación y docencia para la solución de problemas ambientales actuales, y la realización de programas y actividades en el ámbito cultural y en la protección del ambiente como elemento de la formación integral (Universidad Pontificia Bolivariana, 2013).

Aunque la institución dimensiona la importancia de la relación hombre-naturaleza, la mayor problemática ambiental se evidencia en las creencias y comportamientos que manifiesta la comunidad en cuanto al uso, ahorro y manejo de materias primas, energía y agua; residuos sólidos y líquidos, y zonas comunes, además del análisis ambiental en los planes de estudio, el nivel de información que circula en torno al tema y el desarrollo de proyectos de investigación e iniciativas de proyección social en materia ambiental.

El presente proyecto, con una mirada interdisciplinaria, analiza el fenómeno desde los componentes técnico, social, pedagógico y comunicacional, para conocer las creencias, valores, actitudes y comportamientos de la población. El diálogo de saberes entre la Ingeniería y las Ciencias Sociales y Humanas amplía aún más el horizonte, para permear 
la cultura ambiental universitaria a través del abordaje de la realidad de manera integral y transversal, la comprensión del pensamiento y comportamiento de estudiantes, docentes y administrativos, la definición de puntos críticos susceptibles para la intervención, y la toma de decisiones para el mejoramiento.

\section{Metodología}

La investigación diseñó diferentes estrategias que aportaron a la documentación teórica, la interpretación de evidencias y la interacción de los investigadores con los actores y su contexto. El contacto con los grupos de interés a través del análisis documental, la revisión de los planes de estudio y las encuestas facilitó la caracterización de la realidad ambiental que dio origen al escenario actual. Los aportes condujeron a la construcción del escenario posible y deseable.

En el presente caso se emplearon las siguientes técnicas:

- Análisis de los documentos institucionales de la Universidad Pontificia Bolivariana que abordan la relación hombre-naturaleza y dimensionan la formación integral a partir de lo ambiental. Se empleó la ficha temática para estudiar las categorías de análisis relacionadas con los componentes técnico, social, pedagógico y comunicacional.

- Revisión de los planes de estudio de los 13 programas académicos de pregrado para la identificación y análisis de temáticas, programas, proyectos de investigación e iniciativas de proyección social en materia ambiental. Con la ficha temática se estudió la categoría relacionada con el componente pedagógico.

- Encuesta dirigida a estudiantes, docentes internos y administrativos para evidenciar las creencias y comportamientos en cuanto al uso, ahorro, manejo de materias primas, energía, agua, recursos naturales y zonas comunes. Asimismo se verificó la existencia de programas, proyectos de investigación e iniciativas de proyección social en materia ambiental, y se diseñó y aplicó un instrumento tipo escala de Likert para medir las categorías relacionadas con los componentes técnico y social. El instrumento contó con 48 ítems distribuidos en cuatro categorías de análisis; se formularon 33 proposiciones de manera positiva y el resto de forma negativa, utilizando en la redacción expresiones claras, un lenguaje al alcance de toda la muestra y evitando tecnicismos. En dicha escala las opciones de respuesta fueron: Muy de acuerdo (5), De acuerdo (4), Ni de acuerdo ni en desacuerdo (3), En desacuerdo (2) y Muy desacuerdo (1).

- Encuesta aplicada a estudiantes, docentes internos y administrativos para conocer su nivel de información con respecto al tema ambiental. Así, se midió la categoría de análisis relacionada con el componente comunicacional, que contempló cinco interrogantes y distintas opciones de respuesta.

Con base en el problema, la pregunta y los objetivos de la investigación se identificaron cuatro categorías de análisis y sus subcategorías. 
Análisis interdisciplinario del estado actual de la cultura ambiental desde los componentes técnico, social...

Tabla 1. Categorías y subcategorías de análisis

\begin{tabular}{|c|l|l|}
\hline No. & \multicolumn{1}{|c|}{ Categorías } & \multicolumn{1}{c|}{ Subcategorías } \\
\hline 1 & Ambiente & Cultura ambiental \\
\hline \multirow{2}{*}{2} & \multirow{2}{*}{ Recursos } & Agua \\
\cline { 3 - 3 } & & Energía \\
\cline { 3 - 3 } & & Aire \\
\hline 3 & Residuos sólidos & Gestión integral \\
\hline 4 & Espacios & Zonas comunes \\
\hline
\end{tabular}

Fuente: Unidad investigativa, 2014

Diseñados los instrumentos se sometieron a juicio de expertos, uno técnico y otro metodológico, para el ajuste de la redacción de las preguntas y la comprensión de las mismas. Por su parte, el marco muestral permitió identificar los elementos de la población objetivo, que se dividió en tres grupos de interés o estratos de muestreo: estudiantes de pregrado, docentes internos y administrativos.

Tabla 2. Estratos de muestreo

\begin{tabular}{|l|c|c|}
\hline \multicolumn{1}{|c|}{ Estrato o Grupo } & Población & Proporción \\
\hline Estudiantes & 1931 & $87,7 \%$ \\
\hline Docentes internos & 92 & $4,2 \%$ \\
\hline Administrativos & 178 & $8,1 \%$ \\
\hline Total 2201 & \multicolumn{2}{|l}{} \\
\hline
\end{tabular}

Fuente: Unidad investigativa, 2014

El tamaño muestral fue probabilístico; todos los elementos de la población tuvieron igual posibilidad de ser seleccionados de acuerdo con el estrato o grupo al que pertenecen. Con un muestreo aleatorio simple estratificado (MAS), el tamaño máximo de muestra se estimó con una confianza del $95 \%$, error de muestreo de 5\% y proporción P = 0,5 en cada estrato. En este sentido, se otorgó mayor tamaño muestral al estrato más grande, para un tamaño de muestra de 327 personas.

Tabla 3. Distribución proporcional por estrato o grupo

\begin{tabular}{|l|c|}
\hline \multicolumn{1}{|c|}{ Estrato o Grupo } & Muestra \\
\hline Estudiantes pregrado & 287 \\
\hline Docentes Internos & 14 \\
\hline Administrativos & 26 \\
\hline Total & 327 \\
\hline
\end{tabular}

Fuente: Unidad investigativa, 2014 
Para la selección de las personas a las que se les aplicó la encuesta se aplicó el método de selección coordinado negativo por cada estrato, para garantizar la aleatoriedad en la selección de los grupos que fueron estudiados.

\section{Resultados}

Los resultados que se presentan a continuación son producto de la interrelación sistemática de los instrumentos y las categorías de estudio. El análisis y la interpretación se hicieron teniendo en cuenta los componentes técnico y social, pedagógico y comunicacional que a continuación se describen:

\section{Componentes técnico y social}

Una vez aplicada la escala de Likert se analizaron e interpretaron los datos. Para tal efecto se expresan los resultados de acuerdo con las categorías de análisis: cultura ambiental, recursos (agua, energía y aire), residuos sólidos (gestión integral) y espacio (zonas comunes).

Para el análisis estadístico se elaboraron tablas de contingencia para los ítems de cada una de las categorías de la escala de Likert por el tipo de vinculación y se calcularon los porcentajes de las opciones de las preguntas dentro de cada tipo de vinculación y en el total de la muestra. El propósito fue observar la percepción por cada tipo de vinculación para identificar si existe diferencia estadística o dependencia en las respuestas; para este fin, se utilizó la prueba chi-cuadrado con una significación de 5 \%. (p-valor<0.05).

Una vez revisadas las 48 tablas de resultados se escogieron 23, que corresponden al 48 \%. Cada tabla especifica las respuestas por tipo de vinculación.

\section{Categoría 1: Cultura ambiental}

Tabla 4. La información que circula en la Universidad Pontificia Bolivariana Montería sobre el uso de los residuos es eficiente.

\begin{tabular}{|c|c|c|c|c|}
\hline \multirow{2}{*}{$\begin{array}{l}\text { Categoríal.Tabla4 } \\
\text { C1.T4 } \\
\text { Estudiante pregrado }\end{array}$} & \multicolumn{3}{|c|}{ Vinculación } & \multirow{2}{*}{ Total } \\
\hline & Administrativo & Docente & & \\
\hline Muy en desacuerdo & $5,9 \%$ & $10,9 \%$ & $14,5 \%$ & $7,8 \%$ \\
\hline En desacuerdo & $27,9 \%$ & $41,8 \%$ & $52,7 \%$ & $33,2 \%$ \\
\hline Ni acuerdo, ni en desacuerdo & $34,8 \%$ & $27,3 \%$ & $30,9 \%$ & $33,2 \%$ \\
\hline De acuerdo & $26,5 \%$ & $18,2 \%$ & $1,8 \%$ & $21,9 \%$ \\
\hline Muy de acuerdo & $4,9 \%$ & $1,8 \%$ & & $3,8 \%$ \\
\hline Total & $100,0 \%$ & $100,0 \%$ & $100,0 \%$ & $100,0 \%$ \\
\hline
\end{tabular}

Fuente: Unidad Investigativa 
Análisis interdisciplinario del estado actual de la cultura ambiental desde los componentes técnico, social...

Tabla 5. Es función exclusiva de los trabajadores del área de servicios generales de la Universidad Pontificia Bolivariana Montería separar los residuos sólidos.

\begin{tabular}{|l|c|c|c|c|}
\hline \multirow{2}{*}{\multicolumn{1}{c|}{$\begin{array}{c}\text { C1.T5 } \\
\text { Estudiante pregrado }\end{array}$}} & \multicolumn{2}{c|}{ Vinculación } & \multirow{2}{*}{ Total } \\
\cline { 2 - 5 } & Administrativo & \multicolumn{2}{c|}{ Docente } & \\
\hline Muy en desacuerdo & $21,6 \%$ & $38,2 \%$ & $40,0 \%$ & $26,4 \%$ \\
\hline En desacuerdo & $44,3 \%$ & $43,6 \%$ & $54,5 \%$ & $45,6 \%$ \\
\hline Ni acuerdo, ni en desacuerdo & $17,4 \%$ & $9,1 \%$ & $3,6 \%$ & $14,4 \%$ \\
\hline De acuerdo & $13,2 \%$ & $5,5 \%$ & & $10,3 \%$ \\
\hline Muy de acuerdo & $3,5 \%$ & $3,6 \%$ & $1,8 \%$ & $3,3 \%$ \\
\hline \multicolumn{1}{|c|}{ Total } & $100,0 \%$ & $100,0 \%$ & $100,0 \%$ & $100,0 \%$ \\
\hline
\end{tabular}

Fuente: Unidad Investigativa, 2015

Tabla 6. La naturaleza se equilibra con el tiempo, por lo tanto, los problemas ambientales los han exagerado.

\begin{tabular}{|c|c|c|c|c|}
\hline \multirow{2}{*}{$\begin{array}{c}\text { C1.T6 } \\
\text { Estudiante pregrado }\end{array}$} & \multicolumn{3}{|c|}{ Vinculación } & \multirow{2}{*}{ Total } \\
\hline & Administrativo & Docente & & \\
\hline Muy en desacuerdo & $35,2 \%$ & $41,8 \%$ & $61,8 \%$ & $39,8 \%$ \\
\hline En desacuerdo & $39,7 \%$ & $41,8 \%$ & $32,7 \%$ & $39,0 \%$ \\
\hline Ni acuerdo, ni en desacuerdo & $10,1 \%$ & $10,9 \%$ & $1,8 \%$ & $9,1 \%$ \\
\hline De acuerdo & $11,1 \%$ & $3,6 \%$ & $3,6 \%$ & $9,1 \%$ \\
\hline Muy de acuerdo & $3,8 \%$ & $1,8 \%$ & & $3,0 \%$ \\
\hline Total & $100,0 \%$ & $100,0 \%$ & $100,0 \%$ & $100,0 \%$ \\
\hline
\end{tabular}

Fuente: Unidad Investigativa 2015

Tabla 7. Las acciones de educación ambiental que llevan a cabo

la Universidad Pontificia Bolivariana Montería solo son útiles para docentes y administrativos.

\begin{tabular}{|c|c|c|c|c|}
\hline \multirow{2}{*}{$\begin{array}{c}\text { C1.T7 } \\
\text { Estudiante pregrado }\end{array}$} & \multicolumn{3}{|c|}{ Vinculación } & \multirow{2}{*}{ Total } \\
\hline & Administrativo & Docente & & \\
\hline Muy en desacuerdo & $10,1 \%$ & $16,4 \%$ & $25,5 \%$ & $13,1 \%$ \\
\hline En desacuerdo & $32,1 \%$ & $38,2 \%$ & $30,9 \%$ & $32,7 \%$ \\
\hline Ni acuerdo, ni en desacuerdo & $33,8 \%$ & $16,4 \%$ & $30,9 \%$ & $31,0 \%$ \\
\hline De acuerdo & $19,2 \%$ & $25,5 \%$ & $12,7 \%$ & $19,1 \%$ \\
\hline Muy de acuerdo & $4,9 \%$ & $3,6 \%$ & & $4,0 \%$ \\
\hline Total & $100,0 \%$ & $100,0 \%$ & $100,0 \%$ & $100,0 \%$ \\
\hline
\end{tabular}

Fuente: Unidad Investigativa 2015 
Tabla 8. La acumulación de basuras no es un problema, ya que se descomponen de forma natural contra tipo vinculación.

\begin{tabular}{|c|c|c|c|c|}
\hline \multirow{2}{*}{$\begin{array}{c}\text { C1. T8. } \\
\text { Estudiante pregrado }\end{array}$} & \multicolumn{3}{|c|}{ Vinculación } & \multirow{2}{*}{ Total } \\
\hline & Administrativo & Docente & & \\
\hline Muy en desacuerdo & $36,6 \%$ & $32,7 \%$ & $41,8 \%$ & $36,8 \%$ \\
\hline En desacuerdo & $41,1 \%$ & $54,5 \%$ & $56,4 \%$ & $45,1 \%$ \\
\hline Ni acuerdo, ni en desacuerdo & $11,1 \%$ & $10,9 \%$ & $1,8 \%$ & $9,8 \%$ \\
\hline De acuerdo & $8,7 \%$ & $1,8 \%$ & & $6,5 \%$ \\
\hline Muy de acuerdo & $2,4 \%$ & & & $1,8 \%$ \\
\hline Total & $100,0 \%$ & $100,0 \%$ & $100,0 \%$ & $100,0 \%$ \\
\hline
\end{tabular}

Fuente: Unidad Investigativa, 2015

Tabla 9. Es necesario mejorar la información en la Universidad Pontificia Bolivariana Montería para generar conciencia de los efectos de las acciones del hombre sobre el ambiente.

\begin{tabular}{|c|c|c|c|c|}
\hline \multirow{2}{*}{\begin{tabular}{c}
\multirow{2}{*}{$\begin{array}{c}\text { C1.T9 } \\
\text { Estudiante pregrado }\end{array}$} \\
\cline { 2 - 5 }
\end{tabular}} & \multicolumn{3}{|c|}{ Vinculación } & \multirow{2}{*}{ Total } \\
\hline En desacuerdo & $2,4 \%$ & & & $1,8 \%$ \\
\hline Ni acuerdo, ni en desacuerdo & $8,4 \%$ & $3,6 \%$ & $3,6 \%$ & $7,1 \%$ \\
\hline De acuerdo & $55,1 \%$ & $52,7 \%$ & $40,0 \%$ & $52,6 \%$ \\
\hline Muy de acuerdo & $34,1 \%$ & $43,6 \%$ & $56,4 \%$ & $38,5 \%$ \\
\hline Total & $100,0 \%$ & $100,0 \%$ & $100,0 \%$ & $100,0 \%$ \\
\hline
\end{tabular}

Fuente: Unidad Investigativa, 2015

Tabla 10. Es mi interés tomar cursos en la Universidad Pontificia Bolivariana Montería relacionados con temas ambientales.

\begin{tabular}{|c|c|c|c|c|}
\hline \multirow{2}{*}{$\begin{array}{c}\text { C1.T10 } \\
\text { Estudiante pregrado }\end{array}$} & \multicolumn{3}{|c|}{ Vinculación } & \multirow{2}{*}{ Total } \\
\hline & Administrativo & Docente & & \\
\hline Muy en desacuerdo & $1,7 \%$ & $1,8 \%$ & $3,6 \%$ & $2,0 \%$ \\
\hline En desacuerdo & $6,6 \%$ & $1,8 \%$ & $1,8 \%$ & $5,3 \%$ \\
\hline Ni acuerdo, ni en desacuerdo & $27,5 \%$ & $9,1 \%$ & $20,0 \%$ & $23,9 \%$ \\
\hline De acuerdo & $45,6 \%$ & $60,0 \%$ & $58,2 \%$ & $49,4 \%$ \\
\hline Muy de acuerdo & $18,5 \%$ & $27,3 \%$ & $16,4 \%$ & $19,4 \%$ \\
\hline Total & $100,0 \%$ & $100,0 \%$ & $100,0 \%$ & $100,0 \%$ \\
\hline
\end{tabular}

Fuente: Unidad Investigativa, 2015 
Análisis interdisciplinario del estado actual de la cultura ambiental desde los componentes técnico, social...

\section{Categoría 2: Recursos}

Tabla 11. Ahorro agua mientras me lavo las manos.

\begin{tabular}{|c|c|c|c|c|}
\hline \multirow{2}{*}{$\begin{array}{c}\text { C2.T11 } \\
\text { Estudiante pregrado }\end{array}$} & \multicolumn{3}{|c|}{ Vinculación } & \multirow{2}{*}{ Total } \\
\hline & Administrativo & Docente & & \\
\hline Muy en desacuerdo & $1,4 \%$ & $1,8 \%$ & $1,8 \%$ & $1,5 \%$ \\
\hline En desacuerdo & $5,9 \%$ & $3,6 \%$ & $1,8 \%$ & $5,0 \%$ \\
\hline Ni acuerdo, ni en desacuerdo & $15,3 \%$ & $3,6 \%$ & $3,6 \%$ & $12,1 \%$ \\
\hline De acuerdo & $55,7 \%$ & $54,5 \%$ & $43,6 \%$ & $53,9 \%$ \\
\hline Muy de acuerdo & $21,6 \%$ & $36,4 \%$ & $49,1 \%$ & $27,5 \%$ \\
\hline Total & $100,0 \%$ & $100,0 \%$ & $100,0 \%$ & $100,0 \%$ \\
\hline
\end{tabular}

Fuente: Unidad Investigativa, 2015

Tabla 12. Ahorro agua mientras me baño.

\begin{tabular}{|c|c|c|c|c|}
\hline \multirow{2}{*}{$\begin{array}{c}\text { C2.T12 } \\
\text { Estudiante pregrado }\end{array}$} & \multicolumn{3}{|c|}{ Vinculación } & \multirow{2}{*}{ Total } \\
\hline & Administrativo & Docente & & \\
\hline Muy en desacuerdo & $2,4 \%$ & $1,8 \%$ & & $2,0 \%$ \\
\hline En desacuerdo & $7,3 \%$ & $1,8 \%$ & $1,8 \%$ & $5,8 \%$ \\
\hline Ni acuerdo, ni en desacuerdo & $20,2 \%$ & $3,6 \%$ & $5,5 \%$ & $15,9 \%$ \\
\hline De acuerdo & $55,7 \%$ & $74,5 \%$ & $63,6 \%$ & $59,4 \%$ \\
\hline Muy de acuerdo & $14,3 \%$ & $18,2 \%$ & $29,1 \%$ & $16,9 \%$ \\
\hline Total & $100,0 \%$ & $100,0 \%$ & $100,0 \%$ & $100,0 \%$ \\
\hline
\end{tabular}

Fuente: Unidad Investigativa, 2015

Tabla 13. Ahorro agua mientras me cepillo los dientes.

\begin{tabular}{|l|c|c|c|c|}
\hline \multirow{2}{*}{\begin{tabular}{c}
\multirow{2}{*}{$\begin{array}{c}\text { C2.T13 } \\
\text { Estudiante pregrado }\end{array}$} \\
\cline { 2 - 4 }
\end{tabular}} & \multicolumn{3}{|c|}{ Vinculación } & \multirow{2}{*}{ Total } \\
\hline Muy en desacuerdo & $1,0 \%$ & & $3,6 \%$ & $1,3 \%$ \\
\hline En desacuerdo & $6,3 \%$ & $5,5 \%$ & $1,8 \%$ & $5,5 \%$ \\
\hline Ni acuerdo, ni en desacuerdo & $13,9 \%$ & $5,5 \%$ & $5,5 \%$ & $11,6 \%$ \\
\hline De acuerdo & $54,0 \%$ & $65,5 \%$ & $49,1 \%$ & $54,9 \%$ \\
\hline Muy de acuerdo & $24,7 \%$ & $23,6 \%$ & $40,0 \%$ & $26,7 \%$ \\
\hline Total & $100,0 \%$ & $100,0 \%$ & $100,0 \%$ & $100,0 \%$ \\
\hline
\end{tabular}

Fuente: Unidad Investigativa, 2015 
Tabla 14. Ahorro energía para disminuir los costos económicos

\begin{tabular}{|c|c|c|c|c|}
\hline \multirow{2}{*}{$\begin{array}{c}\text { C2.T14 } \\
\text { Estudiante pregrado }\end{array}$} & \multicolumn{3}{|c|}{ Vinculación } & \multirow{2}{*}{ Total } \\
\hline & Administrativo & Docente & & \\
\hline Muy en desacuerdo & $3,5 \%$ & $7,3 \%$ & $14,5 \%$ & $5,5 \%$ \\
\hline En desacuerdo & $23,3 \%$ & $34,5 \%$ & $43,6 \%$ & $27,7 \%$ \\
\hline Ni acuerdo, ni en desacuerdo & $27,2 \%$ & $20,0 \%$ & $18,2 \%$ & $24,9 \%$ \\
\hline De acuerdo & $35,2 \%$ & $30,9 \%$ & $12,7 \%$ & $31,5 \%$ \\
\hline Muy de acuerdo & $10,8 \%$ & $7,3 \%$ & $10,9 \%$ & $10,3 \%$ \\
\hline Total & $100,0 \%$ & $100,0 \%$ & $100,0 \%$ & $100,0 \%$ \\
\hline
\end{tabular}

Fuente: Unidad Investigativa, 2015

Tabla 15. Apago luces y ventiladores al salir del salón de clases u oficina porque contribuye a la preservación de los recursos naturales.

\begin{tabular}{|c|c|c|c|c|}
\hline \multirow{2}{*}{$\begin{array}{l}\text { C2.15 } \\
\text { Estudiante pregrado }\end{array}$} & \multicolumn{3}{|c|}{ Vinculación } & \multirow{2}{*}{ Total } \\
\hline & Administrativo & Docente & & \\
\hline Muy en desacuerdo & $3,5 \%$ & & & $2,5 \%$ \\
\hline En desacuerdo & $9,1 \%$ & & & $6,5 \%$ \\
\hline Ni acuerdo, ni en desacuerdo & $20,6 \%$ & $1,8 \%$ & $3,6 \%$ & $15,6 \%$ \\
\hline De acuerdo & $45,6 \%$ & $41,8 \%$ & $25,5 \%$ & $42,3 \%$ \\
\hline Muy de acuerdo & $21,3 \%$ & $56,4 \%$ & $70,9 \%$ & $33,0 \%$ \\
\hline Total & $100,0 \%$ & $100,0 \%$ & $100,0 \%$ & $100,0 \%$ \\
\hline
\end{tabular}

Fuente: Unidad Investigativa, 2015

Tabla 16. Me resulta fácil apagar las luces del aula u oficina cuando no las estoy usando.

\begin{tabular}{|c|c|c|c|c|}
\hline \multirow{2}{*}{$\begin{array}{c}\text { C2.T16 } \\
\text { Estudiante Pregrado }\end{array}$} & \multicolumn{3}{|c|}{ Vinculación } & \multirow{2}{*}{ Total } \\
\hline & Administrativo & Docente & & \\
\hline Muy en desacuerdo & $2,4 \%$ & $3,6 \%$ & $3,6 \%$ & $2,8 \%$ \\
\hline En desacuerdo & $8,7 \%$ & & & $6,3 \%$ \\
\hline Ni acuerdo, ni en desacuerdo & $16,4 \%$ & $3,6 \%$ & $1,8 \%$ & $12,6 \%$ \\
\hline De acuerdo & $52,3 \%$ & $54,5 \%$ & $47,3 \%$ & $51,9 \%$ \\
\hline Muy de acuerdo & $20,2 \%$ & $38,2 \%$ & $47,3 \%$ & $26,4 \%$ \\
\hline Total & $100,0 \%$ & $100,0 \%$ & $100,0 \%$ & $100,0 \%$ \\
\hline
\end{tabular}

Fuente: Unidad Investigativa, 2015 
Análisis interdisciplinario del estado actual de la cultura ambiental desde los componentes técnico, social...

Tabla 17. He recibido información en la Universidad sobre la importancia del uso y ahorro de la energía contra tipo de vinculación.

\begin{tabular}{|l|c|c|c|c|}
\hline \multirow{2}{*}{\multicolumn{1}{c|}{$\begin{array}{c}\text { C2.T17 } \\
\text { Estudiante Pregrado }\end{array}$}} & \multicolumn{3}{|c|}{ Vinculación } & \multirow{2}{*}{ Total } \\
\cline { 2 - 5 } Mudministrativo & Docente & $14,5 \%$ & $10,1 \%$ \\
\hline En desacuerdo & $9,4 \%$ & $9,1 \%$ & $29,1 \%$ & $31,0 \%$ \\
\hline Ni acuerdo, ni en desacuerdo & $27,9 \%$ & $12,7 \%$ & $25,5 \%$ & $25,4 \%$ \\
\hline De acuerdo & $25,1 \%$ & $52,7 \%$ & $30,9 \%$ & $29,7 \%$ \\
\hline Muy de acuerdo & $3,8 \%$ & $7,3 \%$ & & $3,8 \%$ \\
\hline \multicolumn{1}{|c|}{ Total } & $100,0 \%$ & $100,0 \%$ & $100,0 \%$ & $100,0 \%$ \\
\hline
\end{tabular}

Fuente: Unidad Investigativa, 2015

Tabla 18. Tener una sociedad más desarrollada implica tolerar el ruido causado por las innovaciones tecnológicas.

\begin{tabular}{|c|c|c|c|c|}
\hline \multirow{2}{*}{$\begin{array}{c}\text { C2.T18 } \\
\text { Estudiante pregrado }\end{array}$} & \multicolumn{3}{|c|}{ Vinculación } & \multirow{2}{*}{ Total } \\
\hline & Administrativo & Docente & & \\
\hline Muy en desacuerdo & $17,1 \%$ & $21,8 \%$ & $52,7 \%$ & $22,7 \%$ \\
\hline En desacuerdo & $34,1 \%$ & $52,7 \%$ & $41,8 \%$ & $37,8 \%$ \\
\hline Ni acuerdo, ni en desacuerdo & $22,6 \%$ & $12,7 \%$ & & $18,1 \%$ \\
\hline De acuerdo & $19,9 \%$ & $7,3 \%$ & $5,5 \%$ & $16,1 \%$ \\
\hline Muy de acuerdo & $6,3 \%$ & $5,5 \%$ & & $5,3 \%$ \\
\hline Total & $100,0 \%$ & $100,0 \%$ & $100,0 \%$ & $100,0 \%$ \\
\hline
\end{tabular}

Fuente: Unidad Investigativa, 2015

Tabla 19. Los beneficios de los productos de consumo son más importantes que la contaminación que resulta de su producción.

\begin{tabular}{|c|c|c|c|c|}
\hline \multirow{2}{*}{$\begin{array}{c}\text { C2.T19 } \\
\text { Estudiante pregrado }\end{array}$} & \multicolumn{3}{|c|}{ Vinculación } & \multirow{2}{*}{ Total } \\
\hline & Administrativo & Docente & & \\
\hline Muy en desacuerdo & $18,5 \%$ & $25,5 \%$ & $36,4 \%$ & $21,9 \%$ \\
\hline En desacuerdo & $40,8 \%$ & $52,7 \%$ & $56,4 \%$ & $44,6 \%$ \\
\hline Ni acuerdo, ni en desacuerdo & $22,3 \%$ & $14,5 \%$ & $3,6 \%$ & $18,6 \%$ \\
\hline De acuerdo & $15,3 \%$ & $7,3 \%$ & $3,6 \%$ & $12,6 \%$ \\
\hline Muy de acuerdo & $3,1 \%$ & & & $2,3 \%$ \\
\hline Total & $100,0 \%$ & $100,0 \%$ & $100,0 \%$ & $100,0 \%$ \\
\hline
\end{tabular}

Fuente: Unidad Investigativa, 2015 


\section{Categoría 3: Residuos sólidos}

Tabla 20. Prefiero darle un segundo uso a las cosas antes de tirarlas a la basura.

\begin{tabular}{|l|c|c|c|c|}
\hline \multirow{2}{*}{$\begin{array}{c}\text { C3.T20 } \\
\text { Estudiante pregrado }\end{array}$} & \multicolumn{3}{|c|}{ Vinculación } & \multirow{2}{*}{ Total } \\
\cline { 2 - 4 } & Administrativo & Docente & \\
\hline Muy en desacuerdo & $1,7 \%$ & $1,8 \%$ & $3,6 \%$ & $2,0 \%$ \\
\hline En desacuerdo & $11,8 \%$ & $9,1 \%$ & $3,6 \%$ & $10,3 \%$ \\
\hline Ni acuerdo, ni en desacuerdo & $34,8 \%$ & $18,2 \%$ & $16,4 \%$ & $30,0 \%$ \\
\hline De acuerdo & $41,5 \%$ & $52,7 \%$ & $52,7 \%$ & $44,6 \%$ \\
\hline Muy de acuerdo & $10,1 \%$ & $18,2 \%$ & $23,6 \%$ & $13,1 \%$ \\
\hline Total & $100,0 \%$ & $100,0 \%$ & $100,0 \%$ & $100,0 \%$ \\
\hline
\end{tabular}

Fuente: Unidad Investigativa, 2015

Tabla 21. Cuando compro, selecciono productos elaborados con materiales que se puedan reutilizar o reciclar contra tipo de vinculación.

\begin{tabular}{|c|c|c|c|c|}
\hline \multirow{2}{*}{$\begin{array}{c}\text { C3.T21 } \\
\text { Estudiante pregrado }\end{array}$} & \multicolumn{3}{|c|}{ Vinculación } & \multirow{2}{*}{ Total } \\
\hline & Administrativo & Docente & & \\
\hline Muy en desacuerdo & $3,5 \%$ & $3,6 \%$ & $3,6 \%$ & $3,5 \%$ \\
\hline En desacuerdo & $23,3 \%$ & $7,3 \%$ & $7,3 \%$ & $18,9 \%$ \\
\hline Ni acuerdo, ni en desacuerdo & $43,9 \%$ & $43,6 \%$ & $40,0 \%$ & $43,3 \%$ \\
\hline De acuerdo & $24,0 \%$ & $36,4 \%$ & $45,5 \%$ & $28,7 \%$ \\
\hline Muy de acuerdo & $5,2 \%$ & $9,1 \%$ & $3,6 \%$ & $5,5 \%$ \\
\hline Total & $100,0 \%$ & $100,0 \%$ & $100,0 \%$ & $100,0 \%$ \\
\hline
\end{tabular}

Fuente: Unidad Investigativa, 2015

Tabla 22. En la Universidad, me abstengo de depositar la basura en los recipientes específicos porque están muy distantes de los espacios que frecuento.

\begin{tabular}{|c|c|c|c|c|}
\hline \multirow{2}{*}{$\begin{array}{c}\text { C3.T22 } \\
\text { Estudiante pregrado }\end{array}$} & \multicolumn{3}{|c|}{ Vinculación } & \multirow{2}{*}{ Total } \\
\hline & Administrativo & Docente & & \\
\hline Muy en desacuerdo & $15,3 \%$ & $16,4 \%$ & $36,4 \%$ & $18,4 \%$ \\
\hline En desacuerdo & $34,5 \%$ & $45,5 \%$ & $36,4 \%$ & $36,3 \%$ \\
\hline Ni acuerdo, ni en desacuerdo & $25,1 \%$ & $18,2 \%$ & $16,4 \%$ & $22,9 \%$ \\
\hline De acuerdo & $19,2 \%$ & $12,7 \%$ & $7,3 \%$ & $16,6 \%$ \\
\hline Muy de acuerdo & $5,9 \%$ & $7,3 \%$ & $3,6 \%$ & $5,8 \%$ \\
\hline Total & $100,0 \%$ & $100,0 \%$ & $100,0 \%$ & $100,0 \%$ \\
\hline
\end{tabular}

Fuente: Unidad Investigativa, 2015 
Análisis interdisciplinario del estado actual de la cultura ambiental desde los componentes técnico, social...

Tabla 23. Prefiero usar productos desechables porque son higiénicos y prácticos.

\begin{tabular}{|l|c|c|c|c|}
\hline \multirow{2}{*}{$\begin{array}{c}\text { C3.T23 } \\
\text { Estudiante pregrado }\end{array}$} & \multicolumn{3}{|c|}{ Vinculación } & \multirow{2}{*}{ Total } \\
\cline { 2 - 4 } & Administrativo & Docente & \\
\hline Muy en desacuerdo & $4,2 \%$ & $5,5 \%$ & $7,3 \%$ & $4,8 \%$ \\
\hline En desacuerdo & $15,7 \%$ & $34,5 \%$ & $20,0 \%$ & $18,9 \%$ \\
\hline Ni acuerdo, ni en desacuerdo & $36,2 \%$ & $36,4 \%$ & $32,7 \%$ & $35,8 \%$ \\
\hline De acuerdo & $35,9 \%$ & $16,4 \%$ & $36,4 \%$ & $33,2 \%$ \\
\hline Muy de acuerdo & $8,0 \%$ & $7,3 \%$ & $3,6 \%$ & $7,3 \%$ \\
\hline Total & $100,0 \%$ & $100,0 \%$ & $100,0 \%$ & $100,0 \%$ \\
\hline
\end{tabular}

Fuente: Unidad Investigativa, 2015

Tabla 24. Acostumbro a depositar los residuos sólidos en los puntos destinados para tal fin.

\begin{tabular}{|l|c|c|c|c|}
\hline \multirow{2}{*}{$\begin{array}{c}\text { C3.T24 } \\
\text { Estudiante pregrado }\end{array}$} & \multicolumn{3}{|c|}{ Vinculación } & \multirow{2}{*}{ Total } \\
\cline { 2 - 5 } & Administrativo & Docente & \\
\hline Muy en desacuerdo & $1,0 \%$ & $1,8 \%$ & & $1,0 \%$ \\
\hline En desacuerdo & $7,0 \%$ & $7,3 \%$ & & $6,0 \%$ \\
\hline Ni acuerdo, ni en desacuerdo & $19,5 \%$ & $9,1 \%$ & $10,9 \%$ & $16,9 \%$ \\
\hline De acuerdo & $55,4 \%$ & $54,5 \%$ & $56,4 \%$ & $55,4 \%$ \\
\hline Muy de acuerdo & $17,1 \%$ & $27,3 \%$ & $32,7 \%$ & $20,7 \%$ \\
\hline Total & $100,0 \%$ & $100,0 \%$ & $100,0 \%$ & $100,0 \%$ \\
\hline
\end{tabular}

Fuente: Unidad Investigativa, 2015

\section{Categoría 4. Espacio}

Tabla 25. El mantenimiento y conservación de las zonas de esparcimiento de la Universidad son responsabilidad exclusiva del área administrativa.

\begin{tabular}{|l|c|c|c|c|}
\hline \multirow{2}{*}{$\begin{array}{c}\text { C4.T25 } \\
\text { Estudiante pregrado }\end{array}$} & \multicolumn{3}{|c|}{ Vinculación } & \multirow{2}{*}{ Total } \\
\cline { 2 - 5 } & Administrativo & Docente & \\
\hline Muy en desacuerdo & $21,6 \%$ & $36,4 \%$ & $58,2 \%$ & $28,7 \%$ \\
\hline En desacuerdo & $40,4 \%$ & $58,2 \%$ & $32,7 \%$ & $41,8 \%$ \\
\hline Ni acuerdo, ni en desacuerdo & $16,7 \%$ & $5,5 \%$ & $1,8 \%$ & $13,1 \%$ \\
\hline De acuerdo & $17,4 \%$ & & $7,3 \%$ & $13,6 \%$ \\
\hline Muy de acuerdo & $3,8 \%$ & & & $2,8 \%$ \\
\hline Total & $100,0 \%$ & $100,0 \%$ & $100,0 \%$ & $100,0 \%$ \\
\hline
\end{tabular}

Fuente: Unidad Investigativa, 2015 
Tabla 26. La contaminación genera espacios desagradables.

\begin{tabular}{|c|c|c|c|c|}
\hline \multirow{2}{*}{$\begin{array}{c}\text { C4.T26 } \\
\text { Estudiante pregrado }\end{array}$} & \multicolumn{3}{|c|}{ Vinculación } & \multirow{2}{*}{ Total } \\
\hline & Administrativo & Docente & & \\
\hline Muy en desacuerdo & $4,5 \%$ & $7,3 \%$ & & $4,3 \%$ \\
\hline En desacuerdo & $3,1 \%$ & & & $2,3 \%$ \\
\hline Ni acuerdo, ni en desacuerdo & $2,8 \%$ & $3,6 \%$ & & $2,5 \%$ \\
\hline De acuerdo & $27,2 \%$ & $27,3 \%$ & $12,7 \%$ & $25,2 \%$ \\
\hline Muy de acuerdo & $62,4 \%$ & $61,8 \%$ & $87,3 \%$ & $65,7 \%$ \\
\hline Total & $100,0 \%$ & $100,0 \%$ & $100,0 \%$ & $100,0 \%$ \\
\hline
\end{tabular}

Fuente: Unidad investigativa, 2015

\section{COMPONENTE COMUNICACIONAL}

Tabla 27. Medio de comunicación de la Universidad Pontificia Bolivariana Montería por el que recibe la información de lo que sucede internamente.

\begin{tabular}{|l|c|c|c|c|}
\hline \multirow{2}{*}{ T27 } & \multicolumn{3}{|c|}{ Vinculación } & \multirow{2}{*}{ Total } \\
\cline { 2 - 4 } & Estudiante pregrado & Administrativo & Docente & \\
\hline Internet & $74,6 \%$ & $80,0 \%$ & $94,5 \%$ & $78,1 \%$ \\
\hline Prensa & $3,8 \%$ & $3,6 \%$ & $0,0 \%$ & $3,3 \%$ \\
\hline Radio & $2,4 \%$ & $14,5 \%$ & $5,5 \%$ & $4,5 \%$ \\
\hline Televisión & $19,2 \%$ & $1,8 \%$ & $0,0 \%$ & $14,1 \%$ \\
\hline Total & $100,0 \%$ & $100,0 \%$ & $100,0 \%$ & $100,0 \%$ \\
\hline
\end{tabular}

Fuente: Unidad investigativa, 2015

Tabla 28. Manera en que conoce lo que sucede en la universidad.

\begin{tabular}{|l|c|c|c|c|}
\hline \multirow{2}{*}{ T28 } & \multicolumn{3}{|c|}{ Vinculación } & \multirow{2}{*}{ Total } \\
\cline { 2 - 4 } & $\begin{array}{c}\text { Estudiante } \\
\text { pregrado }\end{array}$ & Administrativo & Docente & \\
\hline Evento & $9,4 \%$ & $1,8 \%$ & $0,0 \%$ & $7,1 \%$ \\
\hline Medio de comunicación externa & $9,1 \%$ & $1,8 \%$ & $0,0 \%$ & $6,8 \%$ \\
\hline Medio de comunicación interna & $24,0 \%$ & $90,9 \%$ & $81,8 \%$ & $41,3 \%$ \\
\hline Reuniones & $3,8 \%$ & $5,5 \%$ & $10,9 \%$ & $5,0 \%$ \\
\hline Rumores & $24,7 \%$ & $0,0 \%$ & $1,8 \%$ & $18,1 \%$ \\
\hline $\begin{array}{l}\text { Información proporcionada por } \\
\text { compañero }\end{array}$ & $28,9 \%$ & $0,0 \%$ & $5,5 \%$ & $21,7 \%$ \\
\hline \multicolumn{1}{|c|}{ Total } & $100,0 \%$ & $100,0 \%$ & $100,0 \%$ & $100,0 \%$ \\
\hline
\end{tabular}

Fuente: Unidad investigativa, 2015 
Análisis interdisciplinario del estado actual de la cultura ambiental desde los componentes técnico, social...

Tabla 29. Medio de comunicación por el que prefiere estar informado.

\begin{tabular}{|l|l|l|l|l|}
\hline \multirow{2}{*}{ T29 } & \multicolumn{3}{|c|}{ Vinculación } & \multirow{2}{*}{ Total } \\
\cline { 2 - 4 } & Estudiante pregrado & \multicolumn{1}{|c|}{ Administrativo } & \multicolumn{1}{c|}{ Docente } & \\
\hline Carteleras & $11,1 \%$ & $1,8 \%$ & $5,5 \%$ & $9,1 \%$ \\
\hline Internet & $67,9 \%$ & $72,7 \%$ & $78,2 \%$ & $70,0 \%$ \\
\hline Prensa & $4,5 \%$ & $1,8 \%$ & $5,5 \%$ & $4,3 \%$ \\
\hline Radio & $1,4 \%$ & $16,4 \%$ & $5,5 \%$ & $4,0 \%$ \\
\hline Televisión & $15,0 \%$ & $7,3 \%$ & $5,5 \%$ & $12,6 \%$ \\
\hline \multicolumn{1}{|c|}{ Total } & $100,0 \%$ & $100,0 \%$ & $100,0 \%$ & $100,0 \%$ \\
\hline
\end{tabular}

Fuente: Unidad investigativa, 2015

Tabla 30. Medio de comunicación alternativo por el que prefiere estar informado.

\begin{tabular}{|c|c|c|c|c|}
\hline \multirow{2}{*}{$\mathrm{T} 30$} & \multicolumn{3}{|c|}{ Vinculación } & \multirow{2}{*}{ Total } \\
\hline & Estudiante pregrado & Administrativo & Docente & \\
\hline Campañas pedagógicas & $22,6 \%$ & $54,5 \%$ & $49,1 \%$ & $30,7 \%$ \\
\hline Cartillas & $5,9 \%$ & $7,3 \%$ & $12,7 \%$ & $7,1 \%$ \\
\hline Cine foro & $2,8 \%$ & $1,8 \%$ & $1,8 \%$ & $2,5 \%$ \\
\hline Folleto / Plegable & $29,3 \%$ & $14,5 \%$ & $7,3 \%$ & $24,2 \%$ \\
\hline Fotografía & $18,8 \%$ & $5,5 \%$ & $5,5 \%$ & $15,1 \%$ \\
\hline Puesta en escena & $4,5 \%$ & $3,6 \%$ & $7,3 \%$ & $4,8 \%$ \\
\hline Video & $16,0 \%$ & $12,7 \%$ & $16,4 \%$ & $15,6 \%$ \\
\hline Total & $100,0 \%$ & $100,0 \%$ & $100,0 \%$ & $100,0 \%$ \\
\hline
\end{tabular}

Fuente: Unidad investigativa, 2015

Tabla 31. Credibilidad de la información emitida acerca de la universidad.

\begin{tabular}{|c|c|c|c|c|}
\hline \multirow{2}{*}{ T31 } & \multicolumn{3}{|c|}{ Vinculación } & \multirow{2}{*}{ Total } \\
\cline { 2 - 4 } & Estudiante pregrado & Administrativo & Docente & \\
\hline $\begin{array}{l}\text { Coordinador/Jefe de área que } \\
\text { lidera la iniciativa }\end{array}$ & $40,4 \%$ & $40,0 \%$ & $43,6 \%$ & $40,8 \%$ \\
\hline Medio de comunicación & $33,4 \%$ & $38,2 \%$ & $29,1 \%$ & $33,5 \%$ \\
\hline Vocero de la institución & $26,1 \%$ & $21,8 \%$ & $27,3 \%$ & $25,7 \%$ \\
\hline \multicolumn{1}{|c|}{ Total } & $100,0 \%$ & $100,0 \%$ & $100,0 \%$ & $100,0 \%$ \\
\hline
\end{tabular}

Fuente: Unidad investigativa, 2015 


\section{COMPONENTE PEDAGÓGICO}

Por medio de la técnica de la revisión documental se identificó el documento institucional, la fecha de publicación y el abordaje de la cultura ambiental desde los planes de estudio. En el cuadro se destacan los principales aportes.

Cuadro 1. Análisis de documentos institucionales de la Universidad Pontificia Bolivariana Montería -UPB- en relación con la cultura ambiental

\begin{tabular}{|c|c|}
\hline $\begin{array}{l}\text { Documentos } \\
\text { Fecha } \\
\text { publicación }\end{array}$ & Aporte a la cultura ambiental \\
\hline \multirow{2}{*}{$\begin{array}{l}\text { Las universidades } \\
\text { según la constitu- } \\
\text { ción Apostólica "Ex } \\
\text { corde Ecclesiae" } \\
\text { del sumo pontífice } \\
\text { Juan Pablo II } \\
\text { Junio } 2013\end{array}$} & $\begin{array}{l}\text { La finalidad de toda universidad católica es hacer que se logre "una pre- } \\
\text { sencia", pública, continúa y universal del pensamiento cristiano en todo } \\
\text { esfuerzo, tendente a promover la cultura superior y formar hombres insignes. } \\
\text { La universidad católica es una comunidad académica que contribuye a la } \\
\text { tutela y desarrollo de la dignidad humana y de la herencia cultural. }\end{array}$ \\
\hline & $\begin{array}{l}\text { Entre los criterios que determinan el valor de una cultura están el significado } \\
\text { de la persona humana, su libertad, dignidad, sentido de responsabilidad } \\
\text { y apertura a la transcendencia. A las instituciones de carácter superior se } \\
\text { les encomienda la tarea de la transmisión y desarrollo de la ciencia, la téc- } \\
\text { nica y la cultura, mediante la docencia y la investigación, contribuyendo al } \\
\text { enriquecimiento del saber humano. }\end{array}$ \\
\hline $\begin{array}{l}\text { Estatutos } \\
\text { Generales } \\
\text { Junio } 1996\end{array}$ & $\begin{array}{l}\text { Entre los objetivos de la Universidad está realizar programas y actividades } \\
\text { en el ámbito cultural y en la protección del medio ambiente, como elemento } \\
\text { de la formación integral para la comunidad universitaria. }\end{array}$ \\
\hline \multirow{6}{*}{$\begin{array}{l}\text { Proyecto Institu- } \\
\text { cional } \\
\text { Marzo } 2004\end{array}$} & $\begin{array}{l}\text { El componente Ambiente y Desarrollo Sostenible establece la dimensión } \\
\text { ambiental como un eje transversal en el quehacer docente e investigativo. } \\
\text { Así, la Universidad determina un compromiso explícito con la gestión } \\
\text { ambiental y el mejoramiento continuo a través de la evaluación de sus } \\
\text { impactos ambientales, la prevención, control y mitigación de los mismos y } \\
\text { el cumplimiento de la legislación ambiental. }\end{array}$ \\
\hline & $\begin{array}{l}\text { También contribuye con la construcción de una cultura institucional que } \\
\text { manifieste y practique este compromiso con el ambiente mediante las } \\
\text { siguientes estrategias: }\end{array}$ \\
\hline & - Incorporación progresiva de la dimensión ambiental en los currículos. \\
\hline & $\begin{array}{l}\text { - Generación de procesos interdisciplinarios de investigación y docencia } \\
\text { que aporten a la solución de los problemas ambientales actuales. }\end{array}$ \\
\hline & $\begin{array}{l}\text { - Cooperación interinstitucional e intersectorial mediante la transferencia } \\
\text { de conocimiento para el desarrollo de programas, proyectos y planes } \\
\text { que tengan de prioridad el mejoramiento ambiental. }\end{array}$ \\
\hline & $\begin{array}{l}\text { - Fortalecimiento de los programas de postgrado en ciencias del ambiente } \\
\text { y creación de nuevos estudios de formación avanzada en esta área. }\end{array}$ \\
\hline
\end{tabular}


Análisis interdisciplinario del estado actual de la cultura ambiental desde los componentes técnico, social...

\begin{tabular}{|c|c|}
\hline $\begin{array}{l}\text { Documentos } \\
\text { Fecha } \\
\text { publicación }\end{array}$ & Aporte a la cultura ambiental \\
\hline $\begin{array}{l}\text { Régimen Docente } \\
\text { Abril } 2002\end{array}$ & $\begin{array}{l}\text { Este Régimen organiza la carrera profesional en el nivel universitario de } \\
\text { acuerdo con los objetivos de la UPB, estimulando la promoción integral } \\
\text { del profesorado con miras a adecuar cada vez más el nivel académico de } \\
\text { la Universidad a las necesidades sociales y culturales del país, entre ellas, } \\
\text { las ambientales. }\end{array}$ \\
\hline $\begin{array}{l}\text { Régimen Discente } \\
\text { Noviembre } 2003\end{array}$ & $\begin{array}{l}\text { La Universidad establece en su misión la formación integral de las personas } \\
\text { que la constituyen, mediante la evangelización de la cultura, en la búsqueda } \\
\text { constante de la verdad, con procesos de docencia, investigación y servicios } \\
\text { (que aporten, entre otros temas, al ambiental). }\end{array}$ \\
\hline $\begin{array}{l}\text { Código del Buen } \\
\text { Gobierno } \\
\text { Diciembre } 2009\end{array}$ & $\begin{array}{l}\text { El componente Ambiente y Desarrollo Sostenible establece la dimensión } \\
\text { ambiental como un eje transversal en el quehacer docente e investigativo. } \\
\text { Así, la Universidad determina un compromiso explícito con la gestión } \\
\text { ambiental y el mejoramiento continuo a través de la evaluación de sus } \\
\text { impactos ambientales, la prevención, control y mitigación de los mismos y } \\
\text { el cumplimiento de la legislación ambiental. }\end{array}$ \\
\hline $\begin{array}{l}\text { Espíritu Bolivariano } \\
2010\end{array}$ & $\begin{array}{l}\text { No se podrá ser un perfecto Bolivariano sin estar convencido de la misión } \\
\text { cultural y social que la Universidad corresponde llenar en la vida colombiana. }\end{array}$ \\
\hline $\begin{array}{l}\text { Modelo Pedagógi - } \\
\text { co Integrado } \\
\text { Octubre } 2009\end{array}$ & $\begin{array}{l}\text { La misión institucional se evidencia en la búsqueda de la verdad por medio } \\
\text { de la investigación y en la evangelización de la cultura a través de los valo- } \\
\text { res institucionales. El docente utiliza métodos para generar un adecuado } \\
\text { ambiente de aprendizaje en el que los estudiantes comparten elementos } \\
\text { culturales, lenguajes, códigos y saberes diversos. }\end{array}$ \\
\hline $\begin{array}{l}\text { Plan de Internacio- } \\
\text { nalización 2013- } \\
2015 \\
\text { Octubre de } 2012\end{array}$ & $\begin{array}{l}\text { La misión de la Universidad considera: "Con la colaboración local e inter- } \\
\text { nacional se logra el intercambio y el enriquecimiento cultural y científico". } \\
\text { La institución "piensa que el desarrollo de la Educación Superior en el } \\
\text { mundo confirma la importancia de extender su proyecto educativo a otras } \\
\text { regiones del departamento, del país y del entorno internacional cercano, } \\
\text { para contribuir a su desarrollo cultural y social". } \\
\text { "La UPB reconoce que las culturas hoy son un entramado complejo, que } \\
\text { se comunican y diferencian a través de sus múltiples manifestaciones, } \\
\text { implicando modos diversos de ver el mundo y practicar el conocimiento". }\end{array}$ \\
\hline $\begin{array}{l}\text { Fundamentos, } \\
\text { definición } \\
\text { y políticas de la } \\
\text { proyección social } \\
\text { Octubre } 2009\end{array}$ & $\begin{array}{l}\text { "La proyección social se enmarca dentro de la Misión en referencia a la } \\
\text { formación integral de profesionales a través de la asimilación, producción, } \\
\text { aplicación y difusión de conocimiento científico, humanístico, tecnológico, } \\
\text { artístico y cultural". Lo anterior tiene como referente a la Constitución Co- } \\
\text { lombiana en su artículo 67, el cual propende por el acceso al conocimiento, } \\
\text { a la ciencia, a la técnica y a los demás bienes y valores de la cultura. }\end{array}$ \\
\hline
\end{tabular}




\begin{tabular}{|c|c|}
\hline $\begin{array}{l}\text { Documentos } \\
\text { Fecha } \\
\text { publicación }\end{array}$ & Aporte a la cultura ambiental \\
\hline $\begin{array}{l}\text { Plan de Desarrollo } \\
\text { 2012-2013 } \\
\text { Diciembre } 2011\end{array}$ & $\begin{array}{l}\text { Entre los objetivos institucionales se encuentra realizar programas y acti- } \\
\text { vidades en el ámbito cultural y el la protección del medio ambiente, como } \\
\text { elemento de la formación integral para la comunidad universitaria. }\end{array}$ \\
\hline $\begin{array}{l}\text { Resolución } \\
\text { Rectoral } 13 \\
\text { Febrero de } 2013\end{array}$ & $\begin{array}{l}\text { La política y el modelo de responsabilidad social compromete a los inte- } \\
\text { grantes de la Universidad; cada uno con su accionar diario está llamado a } \\
\text { desarrollar una gestión institucional que, por su filosofía y naturaleza, es } \\
\text { responsable en lo social, lo económico y lo ambiental. } \\
\text { Así, se espera contribuir en tener un ambiente de calidad, para que las } \\
\text { acciones propias del calentamiento global y otros efectos que se han des- } \\
\text { prendido del uso irresponsables de los recursos naturales, dejen de ser una } \\
\text { amenaza para la generación presente y futura. }\end{array}$ \\
\hline $\begin{array}{l}\text { Plan de Desarrollo } \\
\text { Institucional Sec- } \\
\text { cional Montería, } \\
\text { 2014-2016. } \\
\text { Diciembre } 2013\end{array}$ & $\begin{array}{l}\text { Dentro de los objetivos estratégicos y prospectivos se encuentran la Cali- } \\
\text { dad, Autorregulación y Pertinencia que plantean el establecimiento de un } \\
\text { Sistema de Gestión de Garantía de la Calidad para aunar esfuerzos con el } \\
\text { fin de desarrollar una cultura institucional de calidad. } \\
\text { Una de las líneas estratégicas asociadas a los macroprocesos de la institu- } \\
\text { ción es fomentary participar en la transformación socio-económica, cultural } \\
\text { y política de la región a través del humanismo cristiano. }\end{array}$ \\
\hline $\begin{array}{l}\text { Política de Respon- } \\
\text { sabilidad Social } \\
\text { Febrero } 2013\end{array}$ & $\begin{array}{l}\text { Dentro de las líneas de actuación de la Política de Responsabilidad Social se } \\
\text { encuentra un medio ambiente de calidad, con el fin de aportar con acciones } \\
\text { que contribuyan para que el calentamiento global y otros efectos que se } \\
\text { han desprendido del uso irresponsable de los recursos naturales, dejen de } \\
\text { ser una amenaza para la humanidad presente y futura. } \\
\text { En esta medida, la institución evidencia su compromiso con el desarrollo } \\
\text { sustentable que es exigido globalmente por las comunidades y quienes } \\
\text { lideran las iniciativas que propenden por la buena salud del planeta como } \\
\text { condición necesaria para que la humanidad pueda sobrevivir. }\end{array}$ \\
\hline
\end{tabular}

Fuente: Unidad Investigativa, 2015

En el proceso de revisión de los planes de estudio de los 13 programas de pregrado que ofrece la Universidad Pontificia Bolivariana Seccional Montería, entre los que se encuentran: Comunicación Social-Periodismo, Psicología, Derecho, Administración de Empresas, Economía, Arquitectura, Ingeniería Civil, Ingeniería Mecánica, Ingeniería Electrónica, Ingeniería Industrial, Ingeniería Agroindustrial, Ingeniería Sanitaria y Ambiental e Ingeniería Informática, se identificó que cinco programas ofrecen cursos que se relacionan con el ambiente, lo que se debe a la especificidad de la disciplina y no a la construcción de una cultura ambiental institucional. 
Análisis interdisciplinario del estado actual de la cultura ambiental desde los componentes técnico, social...

Cuadro 2. Cursos que se ofertan desde los programas académicos.

\begin{tabular}{|c|c|c|}
\hline Escuela & Programa & Curso \\
\hline Derecho & Derecho & Derecho Ambiental \\
\hline \multirow{2}{*}{$\begin{array}{l}\text { Ciencias } \\
\text { Sociales y } \\
\text { Humanos }\end{array}$} & \multirow{2}{*}{$\begin{array}{l}\text { Comunicación Social - } \\
\text { Periodismo }\end{array}$} & Comunicación para el Desarrollo I \\
\hline & & Comunicación para el Desarrollo II \\
\hline \multirow{12}{*}{$\begin{array}{l}\text { Ingenierías y } \\
\text { Arquitectura }\end{array}$} & Arquitectura & Bioclimática y Urbanismo \\
\hline & Ingeniería Agroindustrial & Biología y Balance de Materia y Energía \\
\hline & \multirow{10}{*}{ Ingeniería Sanitaria y ambiental } & Ecología \\
\hline & & Geología Ambiental \\
\hline & & Estudios de Impacto Ambiental \\
\hline & & Sistema de Tratamiento del Agua I \\
\hline & & Sistema de Tratamiento del Agua II \\
\hline & & Gestión Ambiental \\
\hline & & Residuos Sólidos \\
\hline & & Saneamiento Ambiental \\
\hline & & Sistema de Saneamiento de Agua Residual \\
\hline & & Contaminación Atmosférica \\
\hline
\end{tabular}

Fuente: Unidad Investigativa, 2015

Cuadro 3. Otras ofertas desde las Unidades Académicas.

\begin{tabular}{|l|l|l|}
\hline \multicolumn{1}{|c|}{ Unidad } & \multicolumn{1}{c|}{ Programa } & \multicolumn{1}{c|}{ Descripción } \\
\hline $\begin{array}{l}\text { Centro de Formación } \\
\text { Humanista }\end{array}$ & Interdisciplinario & Curso Ecología en la Cultura \\
\hline Bienestar Universitario & Interdisciplinario & Comité Interdisciplinario Ambiental \\
\hline $\begin{array}{l}\text { Comunicación Social - } \\
\text { Periodismo }\end{array}$ & Proyección social & $\begin{array}{l}\text { Proyecto Reciclando Ando: la nueva cultura del } \\
\text { reciclaje }\end{array}$ \\
\hline
\end{tabular}

Fuente: Unidad Investigativa, 2015

Además, la Universidad ha liderado el proyecto Reciclando Ando: la nueva cultura del reciclaje en la ciudad de Montería, iniciativa de proyección social creada y gestionada por el programa de Comunicación Social-Periodismo desde el año 2008 con el fin de promover la importancia del ciudadano como agente de cambio, el uso de la comunicación y los medios para la libertad de expresión, la reflexión en torno a las necesidades y retos de la comunidad y el cuidado del medio ambiente a través del tratamiento adecuado de los residuos sólidos. 
Por ocho años consecutivos, se ha beneficiado a 160 líderes y madres comunitarias de nueve barrios, 190 estudiantes de ocho instituciones educativas oficiales, un centro social y una fundación. Solo existe un proyecto de este tipo en la institución, que a pesar de tener un fuerte componente ambiental, no es liderado por el Programa de Ingeniería Sanitaria y Ambiental.

\section{Conclusiones}

- Existe una disonancia entre el conocimiento ambiental que tienen los estudiantes de pregrado, docentes, administrativos y su forma de actuación en el contexto universitario; no hay correspondencia entre el pensamiento y el comportamiento de la comunidad universitaria con respecto al tema ambiental. A pesar de que conocen la importancia y uso de los recursos, residuos sólidos y espacios, no se evidencia en el quehacer institucional la práctica generalizada de valores y normas para la consolidación de la cultura ambiental.

- A pesar de que la comunidad académica y administrativa reconoce su grado de responsabilidad en el manejo integral de los residuos sólidos, la consideran función exclusiva del personal de servicios generales de la institución. Se evidencia que aunque el compromiso ambiental es un asunto que compete y compromete a todos los miembros de la organización, en la Universidad falta acción colectiva debido al poco compromiso de los diferentes actores.

- Existe interés por el ambiente; más aún, cuando el personal considera que la institución no es ajena a esta problemática que afecta a la sociedad actual. Llama la atención la indiferencia de los estudiantes; en sus respuestas no toman una posición definida frente al tema. No obstante, se destaca la necesidad y urgencia de desarrollar iniciativas de cambio en lo que respecta a las prácticas inadecuadas que se dan en las zonas comunes, principalmente en las cafeterías, sala de estudio, patio salón, pasillos, salones de clase, baños, entre otros.

- Es fundamental afianzar el componente pedagógico en la Universidad para promover la cultura ambiental. En la actualidad, solo algunos programas académicos de las Escuelas de Derecho, Ciencias Sociales y Humanas, e Ingeniarías y Arquitectura contemplan en sus planes de estudio cursos relacionados con el tema ambiental, a pesar de ser un tema de importancia mundial. Falta permear los currículos con cursos, proyectos, programas, iniciativas y acciones que aporten al desarrollo de la cultura ambiental en la institución y en la sociedad; más aún, cuando existe disposición de docentes y administrativos para tomar cursos de este tipo; se destaca el grado de motivación de estos últimos.

- En cuanto a los recursos de agua y energía, se evidencia una actitud positiva frente al uso adecuado del agua y se asocia principalmente el ahorro de energía con la disminución de costos económicos. Preocupa que solo un grupo de interés reconoce que recibe información respecto al ahorro de energía y reciclaje. Asimismo, el tema 
del ahorro se relaciona principalmente con el factor económico, dejando a un lado la conciencia ambiental que debe generar este tipo de prácticas ciudadanas.

- El tema de los residuos sólidos no es fácil de abordar en la institución; hasta el momento no ha sido posible su gestión integral debido a la falta de políticas y lineamientos en materia ambiental.

- La Universidad ha invertido en la compra de canecas de colores -dispuestas en puntos estratégicos- y ha realizado jornadas de sensibilización e información para su uso adecuado porque se evidencian dificultades en la clasificación y manejo de los diferentes tipos de residuos; a lo anterior, se suma la falta de personal encargado del proceso de separación, clasificación y disposición final. Con respecto al reciclaje, la población no tiene una posición clara frente al tema, motivada por la poca información que tiene y la falta de conciencia ambiental en materia de reducción, reutilización y reaprovechamiento de los residuos sólidos que se producen en la Universidad.

- En lo que respecta al espacio, un porcentaje significativo de la población considera que el mantenimiento y conservación de las zonas comunes no es responsabilidad de todos. Preocupa su comportamiento en lugares de esparcimiento como las cafeterías, sala de estudio y salón de descanso; así como también en pasillos, baños y salones de clase. A pesar de que la institución ha realizado una importante inversión en materia de remodelación, construcción y adecuación de la infraestructura, acompañada de campañas de información y educación, lideradas desde el área de Bienestar Universitario, es notable su pronto deterioro, producto del mal uso del espacio principalmente por parte de los estudiantes de los diferentes programas académicos.

- Existen documentos institucionales que expresan de forma explícita o implícita la declaración de la Universidad Pontificia Bolivariana Seccional Montería en materia ambiental. No obstante, aunque los Estatutos Generales, el Proyecto Institucional, el Modelo Pedagógico Integrado, el Régimen Docente, el Régimen Discente, el Código del Buen Gobierno, el Espíritu Bolivariano, el Plan de Internacionalización, los Fundamentos, definición y políticas de la proyección social, el Plan de Desarrollo 2012-2013, el Plan de Desarrollo Institucional Seccional Montería 2014-2016, son las principales cartas de navegación al respecto, no existe una política ambiental que plantee los lineamientos y directrices para el diseño, implementación y seguimiento de proyectos, programas, iniciativas y acciones que permitan fomentar las actitudes proambientales entre la comunidad universitaria, y por ende, la cultura ambiental.

- El uso de recursos y de espacios, el manejo de los residuos sólidos y la falta de gestión integral son problemáticas que afectan la cultura ambiental; falta generar estrategias ambientales que contrarresten los problemas existentes. Es vital la inclusión de escenarios de participación ciudadana en la construcción del ideal de cultura ambiental para garantizar la participación, concertación e inclusión de los diferentes estamentos como acción estratégica para determinar el ideal de cultura ambiental y de las nuevas pautas de convivencia. 
- Aunque la Universidad cuenta con diferentes medios de comunicación para la difusión de la información, es evidente que estos no son suficientes para facilitar el proceso de recepción, comprensión y difusión de los mensajes que se generan internamente relacionados con lo ambiental, debido a que la población de docentes, administrativos y estudiantes que existe en la actualidad es diversa en cuanto a sus necesidades y gustos. Preocupa que, aunque existen diferentes canales formales de comunicación, se prefieran los mecanismos informales, lo que incide negativamente en el manejo integral, veraz, oportuno y efectivo de la información que se produce y circula.

- Falta claridad en el concepto de la comunicación y en su hacer; la mayoría de esfuerzos se destinan a los medios masivos. La comunicación es empleada y comprendida de manera vertical, lo que limita su uso participativo para promover la cultura ambiental que permee todos los niveles de la organización, desde los procesos de información, sensibilización, promoción y formación.

- Los esfuerzos en materia de comunicación que lleva a cabo la Universidad no sólo deben destinarse al diseño de mensajes ambientales para ser difundidos por los medios masivos actuales; es preciso hacer uso de los medios alternativos, considerados importantes herramientas populares de difusión y comunicación. Si la intención es el fomento de las actitudes proambientales entre la comunidad académica y administrativa, es necesario el uso de la comunicación más allá de lo informativo de los medios.

- En la Universidad, el uso de la comunicación en el tema ambiental está enfocado sólo a los procesos de sensibilización e información, desconociendo las múltiples posibilidades que ésta ofrece. Se está en mora de definir la intención de la comunicación (informar, sensibilizar, motivar, promocionar y formar), para el desarrollo de una estrategia integral que promueva pensamientos y comportamientos proambientales para el cambio social.

- Es fundamental avanzar en el trabajo interdisciplinario entre los programas y unidades académicas para la realización de proyectos, programas e iniciativas que aporten al desarrollo de la cultura ambiental en la institución, para seguir ampliando el panorama de análisis del problema, abordar la realidad de manera integral desde diferentes miradas y saberes, comprender el pensamiento y comportamiento de la comunidad frente a la situación, proponer acciones de mejoramiento, definir estrategias a partir de los componentes técnico, social, pedagógico y comunicacional y construir la política ambiental de la Universidad Pontificia Bolivariana Seccional Montería. La presente investigación es solo el primer avance en el tema. 


\section{Referencias bibliográficas}

Beldarrín, E. (2004). En torno al término cultura. Horizontes. Universidad Católica de Puerto Rico: Puerto Rico.

Bayón, P. E Morejón, A. (2005). Cultura ambiental y la construcción de entornos de reproducción social en Cuba: un reto para el siglo 21. Instituto de Filosofía, Ministerio de Ciencia, Tecnología y Medio Ambiente, 1-6.

Programa de las Naciones Unidas para el medio ambiente. (2005). Programa de las Naciones Unidas para el medio ambiente. Retrieved Febrero 2013, from Declaración de la Conferencia de las Naciones Unidas sobre el Medio Humano: http://www.pnuma.org/docamb/mh1972.php

Universidad Pontificia Bolivariana (2005). Proyecto Institucional. Proyecto Institucional. Con la Ciencia,la Cultura y el Evangelio, para la transformación social y humana. Ed: Universidad Pontificia Bolivariana, Medellín.

Universidad Pontificia Bolivariana (2013). Estatutos Generales de la Universidad. Ed: Universidad Pontificia Bolivariana, Medellín. 
